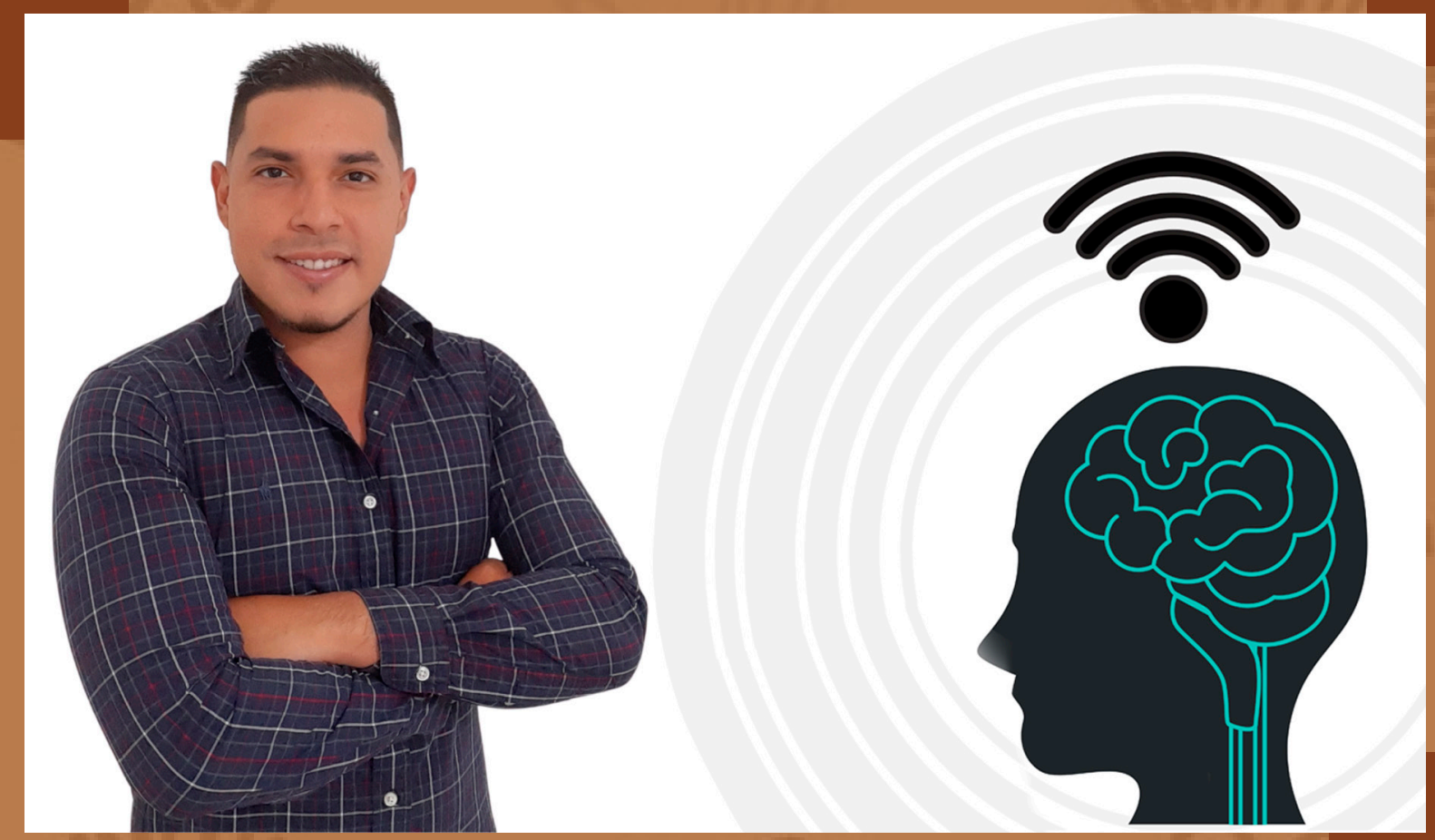

La era digital, el puente alterno en la época de la incertidumbre mundial 


\section{La era digital, el puente alterno en la época de la incertidumbre mundial.}

\section{The digital age, the alternate bridge in the age of global uncertainty.}

Camilo Armando Chavarria Mendoza.

Ingeniero industria y de sistemas organizacionales - Escritor

Recibido: 18-05-2021

https://orcid.org/0000-0003-1108-3983

camilochavarria7489@gmail.com

\section{Resumen}

Los eventos actuales que vive nuestra sociedad han dejado marcado el futuro de las generaciones. La nueva realidad impuso un legado en poco tiempo afectando a todos los puentes de comunicación existentes que con esfuerzo y años de trabajo se edificaron. El ser humano es una entidad cambiante y adaptable a la situación que se le presente, buscará un medio para resolver y saciar sus necesidades, por lo que el cambio en su ambiente es una manera de crecimiento de ideas que prolongarán su estancia en su hábitat natural. Tener el acceso a los medios informativo colmarán de conocimiento a quienes lo posean, pero de igual manera creará un larga brecha que separe a los que carecen de equipos para su obtención. El siguiente estudio refleja de una manera personal las experiencias que están formando de manera trascendental un futuro que no estaba planeado y que ha causado conmoción a nivel mundial, pero que a nivel local aún se sigue luchando para poder adaptarse a los márgenes que se han impuesto para lograr mantenerse firme en la tormenta.

Palabras claves: Sociedad, realidad, ideas, conocimiento, experiencias, necesidades.

\section{Abstract}

The current events that our society lives have marked the future of generations. The new reality imposed a legacy in a short time affecting all the existing communication bridges that with effort and years of work were built. The human being is a changing entity and adaptable to the situation that is presented to him, he will look for a means to solve and satisfy his needs, so the change in his environment is a way of growing ideas that will prolong his stay in his natural habitat. Having access to the media will fill those who possess it with knowledge, but it will also create a long gap that separates those who lack the equipment to obtain it. The following study reflects in a personal way the experiences that are shaping in a transcendental way a future that was not planned and that has caused a global commotion, but that at the local level still struggles to adapt to the margins that have been imposed. to stand firm in the storm.

Keywords: Reality, ideas, knowledge, experiences, needs. 


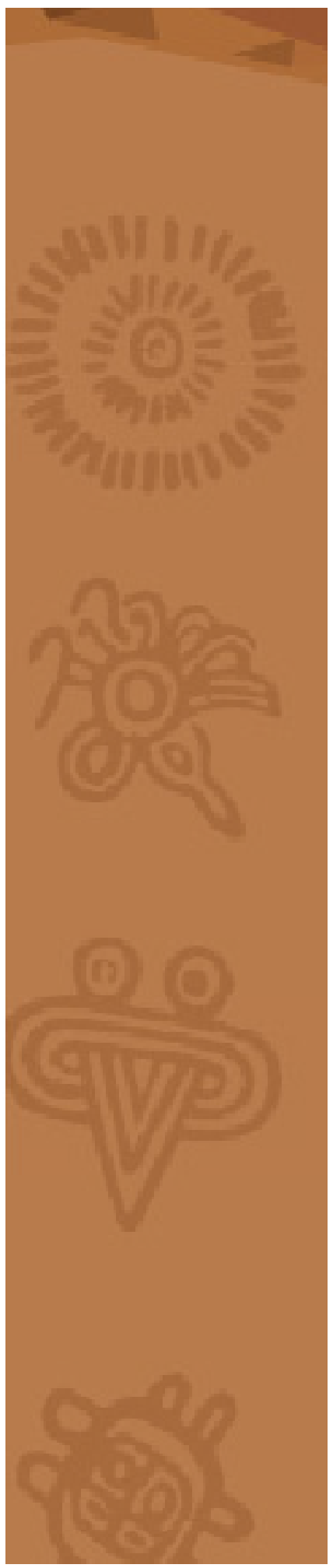

\section{Introducción}

El 2020 dejó un legado histórico que perdurará por muchos siglos, pero no solo de la emergencia sanitaria que doblegó al mundo ocasionando una catástrofe económica mundial, ni de los millones de fallecidos a causa de este devastador capítulo que embriagó a todos los rincones de la tierra, sino, al crecimiento del ser humano ante situaciones contrarias y abrumantes que interfieren con su entorno de su rutina diaria. Una persona normalmente conforme va creciendo en edad, se enfrenta a diferentes situaciones o experiencias que irán formando las decisiones y caracteres que en un momento determinado serán decisivas para alcanzar la estabilidad en un mundo cada vez aglomerado de estereotipos sociales.

El entorno de los países en todos sus etapas esta profundamente arraigado a la educación, extendiéndose desde los primeros pasos hasta lo profesional. Es importante mencionar que los resultados en los estudios superiores están estrechamente relacionados con línea educativa con la cual el individuo se desarrolla desde temprana edad, aunque en el camino puede adquirir herramientas que podrían volcar cualquier habito adquirido.

Las distancias se logran acortar cuando existen vínculos en común dentro de un grupo de personas que buscan construir nuevos caminos en el mundo, siendo el caso de las limitaciones con las cuales fueron puestas obligatoriamente para mitigar la severidad de la situación presente. Por lo cual el ritmo tan agitado que la humanidad vive actualmente y que aborda temas comerciales, políticos y sociales, hicieron que la herramienta del siglo luciera en todo su esplendor, la era tecnológica, liderada por los canales virtuales que han puesto a disposición el estrechamiento de distancias, derribando increíblemente los muros a los cuales estaban en la antigüedad acostumbrados por la falta de información. Es cada vez más fácil lograr mantener vínculos dentro de comunidades, ciudades y países por todo lo largo y ancho del planeta, a tal punto que algunos han llegado a censurar el uso de las redes sociales por dar a conocer información no permitida por las autoridades de cada nación.

En algunos países la pandemia separó a los estudiantes de las escuelas, logrando un duro golpe en el camino de la juventud, por lo que no fue de espera, las plataformas virtuales comenzaron a hacer su hazaña y no permitieron atrasos dentro de la vida estudiantil.

Las aulas se trasladaron a los hogares, y desde una pantalla la información de una clase iba a ser brindada. De manera sublime es un gran avance para evitar un deterioro didáctico, pero de fondo arrastra un sin número de realidades que enfrentan los países en muchas zonas sectorizadas o mejor dicho clasificadas por la economía. No todos tienen las herramientas a sus alcance, por lo que la balanza educativa se inclinará hacia donde exista disponibilidad para lograr lo cometido. 


\section{Desarrollo}

El camino hacia el conocimiento no es fácil y menos cuando tantas corrientes alternas existen, pero la literatura tomó parte importante en todo esto, al lograr traspasar fronteras y colarse dentro de los puentes de comunicación en todo el mundo, dando a conocer la palabra escrita, las nuevas fuentes de ideas y los nuevos precursores de la escritura. De la misma manera otras fuentes emprendedoras empezaron el camino digital hacia el alcance mundial y las aplicaciones sociales no se hicieron esperar para arrebatar por un momento el caos de cada país.

Cada paso que se daba de manera digital entregaba un fuerte avance a la comunicación, al entendimiento y a la distribución de la información. El poder tener al mundo en la palma de la mano es una herramienta perfecta para alzar la voz y dar a conocer cada uno de los ideales. Se necesita criterio para impulsar una idea, pero las redes sociales empoderan a cualquier manera de pensar por lo cual es un arma de doble filo, si no se tiene una mentalidad abierta y tolerante al pensamiento mundial.

Podemos alcanzar lo que queremos, podes realizar las cosas sin mayor esfuerzo, pero cada avance trae consigo nuevas limitantes que se desarrollan fuera del planten de donde emergieron. Juega un papel muy importante la equidad, la igualdad y la empatía, hoy en día cuesta encontrar alguna de estas en la sociedad, pero existen revoluciones que plantean singularidad. En muchos casos el pedir uno de estos tres valores es altamente de escucha fuerte, pero a la vez causa preocupación debido a que todas las corrientes quieren ser escuchadas y las diferencia de criterio causan el caos mediático que al final solo perjudican a los grupos a proteger, causan mayor división y peor aún represalias contra esto. "Esto permite esa mirada de articulación y sintesis, desde el significado de la sociedad digital al uso crítico de las técnicas de observación" (2017). Sociología digital: la reinvención de la investigación social.

Nuestras voces son futuro, son juventud. Donde el poder de la palabra llegue, el incesante fuego del saber detonará en conocimiento perpetuo. De esta manera se ejemplifica el poder de la opinión, es una avalancha que puede hacer una nueva tierra o bien destruir lo que esté a su paso.

Este tipo de temática social últimamente es una corriente alterna donde el principal causante de su origen no es ninguno de los puntos agendados por estos movimientos, si no al excluir pensamientos contrarios crean la guerra de ideas, la cual, al haber una brecha tan grande como la tecnológica hace que el fundamento se pierda llegando a la destructivo y critico sin mediar consecuencias. La facilidad de emplear una opinión sin previo conocimiento de un tema determinado crea confusión, distanciamiento y perjudica grandemente al individuo, no obstante, puede también lograr crear un vinculo cercano al lograr indagar primeramente antes de emitir un mal discurso improvisado.

El ser humano por capacidad propia percibe de manera natural el actuar bien o mal según las normas impuestas por la misma sociedad. El pensar diferentes a estas conlleva a perder en gran medida la libertad en todos sus sentidos. No es muy precipitado aclarar que un sistema se impone para lograr disciplinar a cierto grupo de integrantes para lograr la coordinación y estabilidad necesaria para lograr desarrollar un lugar, una idea o algo que es necesariamente hacerlo de manera conjunta. 


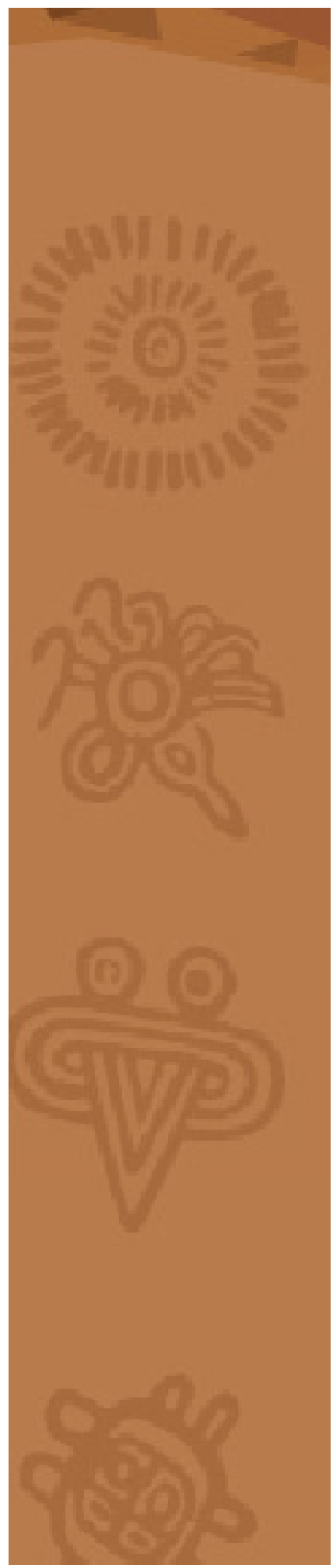

El sistema digital proporcionó un crecimiento sustancial al poder político y económico, dándole un fuerte desplazamiento para dar a conocer sus intereses propios, de igual manera las empresas han ahondado en el correcto manejo de redes para alcanzar un seguimiento de sus respectivas organizaciones y así poder ingresar al tan cotizado mundo mercantil en donde tiene a disposición lograr ser reconocido a niveles internacionales aún trabajando de manera local. Siguiendo con este lineamiento las personas naturales sin un respaldo mediático han logrado dar a conocer algún talento, pasión o pasatiempo que ha valido de distracción para muchos y que hoy se cotiza por cantidad de seguidores, un buen plan de ingresos ante una crisis que a alcanzado a las potencias más grandes del mundo.

Trabajadores que por distancias no logran seguir preparándose educativamente, han logrado continuar con sus estudios gracias a las clases virtuales. Miles de opciones de cursos gratuitos en líneas, seminarios, charlas están a disposición de todos los cuales ayudan a seguir preparándonos ante un presente con mucha incertidumbre y donde el que conoce más lograr tener un escudo mayor como protección de distracciones poco benéficas y que pueden entorpecer el criterio propio.

Escuchamos decir que el poder es querer, en cierta medida tiene mucha razón, vemos a los jóvenes, adultos hasta niños atrapados en un mar virtual que los acompaña las casi las 24 horas del día, donde se predispone un tiempo necesario para adquirir algún tipo de conocimiento. Está pasando que las corrientes que se aglomeran son tantas más la falta de lectura, investigación y la poca énfasis en hacer a la educación como parte de la cultura general, que el interés por superarse no logre ser mayoría ante este gran numero de segmentados y que preocupa que las generaciones que avanzan están logrando abarcar puesto de importancia con nulas habilidades de desempeño y peor aún una empatía inexistente, hasta podría decirse un gozo desenfrenado por rivalidades y competencias poco productivas y alegrías por tragedias ajenas.

El ser humano ha logrado clasificar sus emociones con reacciones generales que son cuantitativas y que te excluyen al ser minoría. Por el contrario, la nube desenfrenada de la mayoría hace tambalear las estructuras de las organizaciones que se han establecido por años y que pueden interferir en los planes de crecimiento regional hasta mundial. La juventud se ha hecho notar a través de sus propuestas vanguardistas que en algunos casos dan un respiro al estar al pendiente de las necesidades de nuestra casa común, punto de estallido global pero que se ve opacado por las nubes grises que denotan la falta de acogimiento a pensamientos ajenos a los picos de popularidad.

Cabe mencionar que la cibercultura tiene un linaje muy sólido en cuanto al desarrollo ligado a la cultura de la computación desde sus inicios y hasta el día de hoy, campo ampliamente fértil para la reflexión antropológica pero que de alguna forma ha sido opacado por conceptos como "cultura digital" "tecnología digital" y el boom recientemente del paradigma anclado en la transformación digital (2020). Antropología Digital: De La Cibercultura a La Transformación Digital. 
La era digital es un puente ante la incertidumbre mundial, en todos los aspectos sociológicos y antropológicos. Todos los seres humanos dependemos de oportunidades que hagan sobresalir o demostrar nuestras capacidades, por lo cual somos tan camaleónicos al momento de querer cumplir una meta, así sea entrar en un nuevo mundo que ofrece variadas opciones para alcanzar dicho sueño. Lo interesante es la respuesta del publico al cambio, hay personas aun renuentes a querer incluirse en este sistema que poco a poco nos ha ido arrastrando a todos y nos mantiene tomados de las manos sin ni siquiera estar de acuerdo.

En el paso del tiempo los grupos sociales se ha ido diversificando por lo cual las características se han ido definiendo de maneras distintas y en el mal uso de la palabra inclusión están dejando entrar cualquier pensamiento sin base y que grita al viento ser escuchado. Recordemos que la libertad de expresión es un derecho del ser humano, pero debe ser acompañado de un criterio bien formado y que conlleve a una idea legitima sin proceder de un anhelo personal.

No podemos crear un movimiento que excluya de manera radical grandes ideas promovidas en la historia, si no, debemos conjugar las mejores propuestas y unificar, porque el suelo que pisamos es el mismo, por lo cual es necesario investigar y valorar cada propuesta con el respeto necesario, ocupando de manera creativa los dispositivos generadores de voz para promulgar una interfaz que podamos entender todos.

\section{Conclusión}

La era digital es el consecutivo de los grandes cambio a nivel mundial que nos ha brindado nuestra propia humanidad, el lograr recrear y establecer nuevas formas de comunicación es un camino a la unidad social, económica y política. Sin embargo, cada cambio debe estar en sintonía con la introducción nivelada en todos los países existentes del mundo, así mismo el acceso a la información y a las herramientas digitales no deben estar solo seleccionada para una parte de la sociedad por lo que lograría un desequilibrio en el crecimiento a gran escala y un desplome de cualquier sistema que se quiera implementar.

El alcance de toda revolución debe ser el cambio en los aspectos en el entorno donde se vive, por lo cual ser agente de cambio con todas las armas para crear un mundo nuevo puede llevar a crear un nuevo planeta donde esté más acorde las posibilidades de todos por mantener una estabilidad donde no existan diferencias y estigmas, donde se celebre con gran jubilo los aspectos propios de cada individuo para mantener un protagonismo digno de una raza que deje huella en el suelo que pisa. 


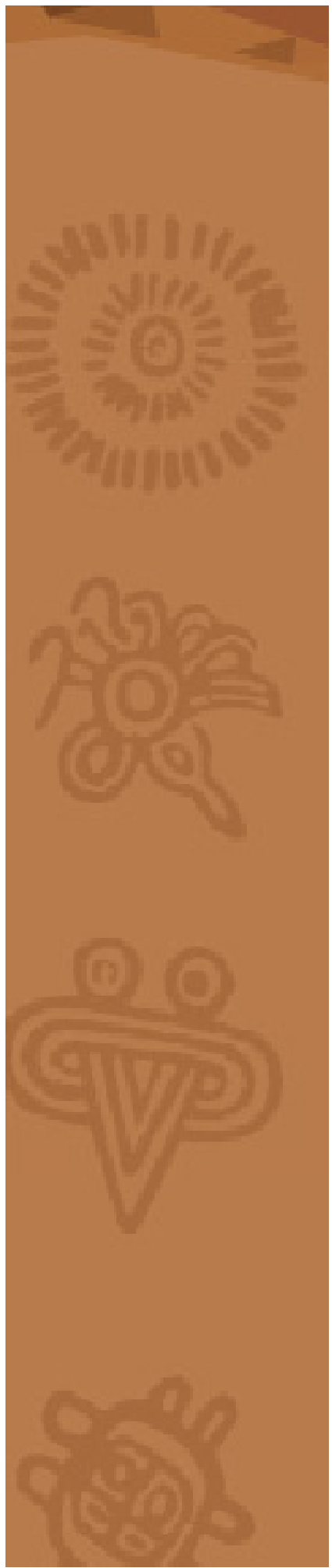

\section{Bibliografía}

Forbes México. (2017). La comunicación humana en la era digital.

Gimena Perret. (2005). Reflexiones antropológicas en la era digital. ¿La empatía amenazada?

Manuel Javier Callejo Gallego. (2017). Sociología digital: la reinvención de la investigación social

Xabier Barandiarán. (2003). La era digital.

Lic. Camilo Armando Chavarria Mendoza.

Egresado de la Universidad Iberoamericana de Ciencia y tecnología UNICIT. Tiene un posgrado en Gerencia de Recursos Humanos. Es uno de los lideres centroamericanos para formar parte del libro "La región que viene: Una mirada a Centroamérica” por el Sistema de Integración Centroamericano (SICA) donde se desarrolla la temática de educación, abarcando los puntos más relevantes en nuestro país y de las regiones sobre situaciones políticas, sociales y económicas que han traído consecuencias al tema. En enero 2021 lanzo de manera digital un cuento corto ilustrado de manera gratuita el cual se titula "La ciudad de los lirios escondidos" en el género de narrativa, logrando clasificar dentro de la aplicación mexicana Ipstory para un lanzamiento formal de manera digital, física y en audio libro. Ha trabajado desde los 19 años en las diferentes áreas de la carrera de ingeniería industrial dentro de las ramas de calidad, recursos humanos, certificaciones internacionales, medio ambiente y actualmente en el área de producción. Se ha desarrollado en diferentes tipos de industrias nacionales y he participado en montaje industrial y productivo con compañías extranjeras. Participación en antologías poéticas y narrativas en diferentes países de Latinoamérica entre ellos: México, Venezuela, Perú, Chile. Ha publicado libros dentro del género de poesía titulado "Casas Rotas", el cual describe en un poema continuo de 600 versos de estrofas de 4 versos de poesía libertaria. Otra obra es "Diario de un febrero bisiesto: 29 poemas de amor y locura" bajo el sello de Sweick book editorial de Chile, el cual tiene una temática romántica en donde describe de manera enumerada una historia de amor, sufrimiento en cada uno de los días que corresponden a un febrero bisiesto. 